\title{
A Standardized Approach to Orthotopic (Life- Supporting) Porcine Cardiac Xenotransplantation in a Non-Human Primate Model
}

\section{Corbin E. Goerlich}

University of Maryland School of Medicine

\section{Bartley P. Griffith}

University of Maryland School of Medicine

John A. Treffalls

University of Maryland School of Medicine

Tianshu Zhang

University of Maryland School of Medicine

Avneesh K. Singh

University of Maryland School of Medicine

\section{Billeta Lewis}

University of Maryland School of Medicine

Ivan Tatarov

University of Maryland School of Medicine

Alena Hershfeld

University of Maryland School of Medicine

\section{Faith Sentz}

University of Maryland School of Medicine

\section{Gheorghe Braileanu}

University of Maryland School of Medicine

\section{David Ayares}

Revivicor, Inc

Muhammad M. Mohiuddin ( $\sim$ MMohiuddin@som.umaryland.edu )

University of Maryland School of Medicine

\section{Research Article}

Keywords: Orthotopic, Porcine Cardiac Xenotransplantation, Non-Human Primate Model, Life-Supporting, Standardized Approach

Posted Date: December 15th, 2021 
DOI: https://doi.org/10.21203/rs.3.rs-1138842/v1

License: (c) (1) This work is licensed under a Creative Commons Attribution 4.0 International License. Read Full License 


\section{Abstract}

There are 5.7 million people in the United States with heart failure, which is life-limiting in $20 \%$ of patients. ${ }^{1}$ While data is most robust in the United States for this cohort, it is known to be a global problem with over 23 million people carrying the diagnosis. ${ }^{1}$ For end-stage heart failure, many require a heart transplantation, however, there is a shortage in the supply of organ donors. Cardiac xenotransplantation has been proposed to "bridge the gap" in supply for these patients requiring transplantation. Recent preclinical success using genetically modified pig donors in baboon recipients has demonstrated survival greater than 6 months. ${ }^{2-5}$ First-in-human transplantation of a genetically modified pig kidney demonstrated 54 hour rejection-free function when perfused by a deceased human recipient, demonstrating the feasibility of cross-species transplantation and invigorating enthusiasm further to utilize this new organ source for a population that would otherwise die waiting for a human organ. ${ }^{6}$ While this human study demonstrated proof-of-principle of overcoming hyperacute rejection, further regulatory oversight by Food and Drug Administration (FDA) may be required with pre-clinical trials in large animal models of xenotransplantation with long-term survival. These studies not only require a multi-disciplinary team and expertise in orthotopic transplantation (cardiac surgery, anesthesia and cardiopulmonary bypass), immunology and genetic engineering; but also, specifically handling large animal recipients that cannot communicate their symptoms. Here we detail our approach to pig-to-primate large animal model of orthotopic cardiac xenotransplantation perioperatively and in the months thereafter in long-term surviving animals. We also detail xenograft surveillance methods and common issues that arise in the postoperative period specific to this model and ways to overcome them.

\section{Introduction:}

The use of cardiac xenotransplantation has been proposed for patients with end-stage heart failure requiring transplantation, but without the means or qualifications to obtain an organ due to shortage of supply. Life-supporting xenograft function in the preclinical setting has been obtained for up to 9 months in a model of cardiac xenotransplantation, achieving preclinical efficacy required for human clinical trials. $^{2-4,7}$ First-in-human transplantation of a genetically modified pig kidney demonstrated 54 hour rejection-free function when perfused by a deceased human recipient, validating anticipated avoidance of hyperacute rejection in pig-to-human transplantation, and further invigorating enthusiasm to utilize this new organ source for a population that would otherwise die waiting for a human organ. ${ }^{6}$ While this study demonstrated proof-of-principle of overcoming hyperacute rejection, further Food and Drug Administration (FDA)-monitored pre-clinical work in large animal models of xenotransplantation may be required using Good Laboratory Practices (GLP) to support an FDA supported human clinical trial. ${ }^{8}$

Preclinical studies in pig-to-baboon cardiac xenotransplantation models require a highly specialized team consisting of researchers, surgeons, anesthesiologists, critical care physicians, veterinary staff, clinical nurses and other laboratory support. Moreover, there are numerous surgical and postoperative care nuances that are critical to the success of this model. We have developed a protocolized approach to this 
model, incorporating our experience of failures but also successes in obtaining the longest surviving preclinical model of orthotopic, life-supporting cardiac xenotransplantation. In this paper we describe 1) the surgical procurement and implantation of pig hearts into non-human primates (NHPs) in the orthotopic position, considering nuances in pig and NHP anatomy, anesthesia and cardiopulmonary bypass, 2) the medical and physical management of these animals in the immediate postoperative period and in the months thereafter, 3) postoperative surveillance of the cardiac xenograft, and 4) common problems and associated solutions. Our goal is to disseminate this information in the transplantation field, empower other laboratories to learn from our experience and to highlight potential management principles relevant to clinical translation that are unique to caring for NHP recipients of a transplanted genetically modified porcine heart xenograft. All procedures described here have been approved by the Institutional Animal Care and Use Committee (IACUC) at the University of Maryland School of Medicine. All methods were carried out in accordance with relevant guidelines and regulations. The reporting in the manuscript follows the recommendations in the ARRIVE guidelines. ${ }^{9}$

\section{Methods:}

\section{Selection of Donor and Recipient for Surgery}

Recipients are selected for low preoperative recipient titers for preformed antibodies ${ }^{10}$ toward xenografts and adequate organ function as measured by a complete metabolic profile. Animals are also acclimated to the jacket, tether and caging system and removed as potential recipients if they fail acclimatization. Donors are weight-matched to the recipient and selected for normal biventricular function without valvular abnormalities, septal defects or pulmonary hypertension. Donors within $10 \%$ of recipient body weight are typically acceptable.

\section{Preparation of the Donor or Recipient for Surgery}

The animal is first sedated to facilitate transport from the holding facility to the operating suite. The sedation is dependent on the animal. A pig is sedated with ketamine $10 \mathrm{mg} / \mathrm{kg}$ and xylazine $2 \mathrm{mg} / \mathrm{kg}$ intramuscularly (I.M.), whereas a non-human primate is sedated using just $10 \mathrm{mg} / \mathrm{kg}$ ketamine. ${ }^{11}$ Prior to entering the operating room, the animal is shaved with veterinarian hair clippers liberally on the chest, neck, abdomen, back and legs. The animal is then scrubbed with chlorohexidine $4.0 \%$, diluted $1: 4$ in all shaved areas and additionally on the hooves or hands and feet. The animal is then transferred to the operating room, where it is prepped and draped widely over the relevant operative field.

\section{Preoperative Placement of the Tunneled Central Line in the Baboon Recipient.}

The placement of a tunneled central line is required to administer medications in the perioperative period, including induction immunosuppression. The recipient is positioned in a partial left lateral decubitus position with a shoulder roll, to maximize the right lateral neck operative field. The right internal jugular vein is the optimal placement for the tunneled central line, however, the left internal jugular vein can also 
be used. The arms are fastened inferiorly, to move the shoulders inferiorly and expose the lateral neck further.

The baboon is prepped and draped in a sterile fashion and a 5 centimeter $(\mathrm{cm})$ incision is made $1 \mathrm{~cm}$ medial and parallel to the medial border of the sternocleidomastoid (SCM). The platysma is exposed and divided with blunt dissection followed by retraction of the SCM laterally. At this layer, the external jugular vein can sometimes be seen. This is distinguished from the internal jugular vein, as it is more superficial and is not contained with a carotid sheath, an adjacent carotid artery or vagus nerve. Fascial layers are carefully divided and small bridging veins tied and ligated down to the carotid sheath. The carotid sheath is incised, the internal jugular vein is separated from the vagus nerve and carotid sheath and proximal and distal control is obtained with 2-0 silk ties.

Attention is then turned to tunneling the line and sizing the catheter. We prefer a 10Fr triple lumen Hickman ( central line (Bard Access Systems, Inc. Salt Lake City, Utah USA, Ref \# 0606560) which comes with a plastic "tunneler", but any rigid narrow rod that can be manipulated under the subcutaneous plane of the animal will suffice. The tunneler is pushed from medial to lateral, starting from the subcutaneous plane in the lateral edge of the neck incision and tunneled toward an exit site mid-point between the scapulae. The central line is then attached to the tunneler sterilely and pulled through the freshly made tract. If the line is cuffed, the line should pull through the skin and the cuff should lie subcutaneously. The distal portion of the line with infusion ports is now exiting the back and the proximal portion of the line is in the surgical neck incision, with a subcutaneous track connecting the two points. The central line is trimmed to allow the tip of the catheter to end at the cavoatrial junction, which is about the right 5th intercostal space at the costosternal junction. Fluoroscopy has been performed by us in the past to trim the catheter more accurately to length in heterotopic experiments, but in orthotopic cases the catheter is trimmed to size during recipient cardiectomy.

When the catheter is appropriately tunneled and sized, the distal (superior) internal jugular vein is ligated, a generous venotomy is performed and the catheter is introduced proximally. The central line is then fastened proximally with the proximal 2-0 silk tie to ensure hemostasis. Several 2-0 silk sutures are tied along the length of the catheter in the neck to create a general inverted " $U$ " loop to prevent line kinking with turning of the neck. Three silicon beads are put on the catheter before its insertion into the jugular vein. These beads are then secured to the fascia with silk sutures. This prevents lateral movement and kinking of the catheter with neck movement. The incision is then closed in several layers, with an emphasis on a fascial closure over the length of the catheter where possible to prevent catheter infection.

\section{Procurement of the Donor Heart.}

Donor cardiac xenografts are procured from genetically modified pigs with multiple carbohydrate knockouts and knockins for human thromboregulatory proteins, human complement regulatory proteins and human anti-inflammatory proteins in order to render xenografts more immunocompatible for crossspecies transplantation. ${ }^{3}$ Donor heart procurement has been described elsewhere, with international 
consensus guidelines on this topic. ${ }^{12}$ Therefore, principles of heart procurement will not be repeated here, but emphasis in heart procurement to the extent that minimizes ventricular distention and ischemia cannot be understated. In cardiac xenotransplantation, primary graft dysfunction (i.e., perioperative cardiac xenograft dysfunction (PCXD)) can occur in $40-60 \%$ of transplantations and can be reduced with ischemia mitigation during this process. ${ }^{13,14}$ The donor is prepped and draped from the edge of the mandible superiorly, 2-4 cm below the xyphoid process inferiorly and the anterior axillary line laterally. A procurement checklist is provided in table 1.

Several things should be noted with emphasis for our approach to protection of the donor heart in cardiac xenotransplantation with pig donors. Induction of cardioplegia should be performed with blood-based solution, ideally $30 \mathrm{cc} / \mathrm{kg}, 25 \%$ of which is fresh blood procured from the donor's aortic root. ${ }^{15}$ Delivery into the clamped ascending aorta is via a $9 \mathrm{Fr}$ cardioplegia catheter connected to bladder irrigation IV tubing, pressurized with an infusion bag set to $150 \mathrm{mmHg}$. Perfusion pressures are titrated with the bladder irrigation tubing to $20-30 \mathrm{mmHg}$ measured at the root by palpation (table 2). In our experience, this gives the best cardioplegia induction with appropriate arrest. Moreover, the aortic cross clamp is best performed across the arch, from left to right, between right and left innominate arteries, as the donor pig has a bovine arch (carotid and subclavian arteries come off brachiocephalic arteries bilaterally). The right brachiocephalic artery can be simply ligated prior to cross-clamping. Cardiectomy should be planned to minimize ischemic back table preparation prior to implantation. Therefore, we elect to vent the left side, only venting the right side if left or right ventricular distention is observed. If the right ventricle (RV) begins to distend, the SVC should be quickly opened superior to the azygous vein. If the left ventricle (LV) distends, the right pulmonary vein should be opened, cutting widely towards the hilum of the right lung.

\section{Non-Ischemic Continuous Perfusion (NICP) Preservation:}

NICP has been shown to improve outcomes in cardiac xenotransplantation. 2,13,15 If electing to preserve the cardiac xenograft with NICP, a perfusion cannula is inserted into the aortic arch at the level of the right innominate artery. During donor explant, the aorta is transected at the level of the right innominate artery so that if the NICP cannula is too large, the transected aortotomy can be enlarged by incorporating the transected right innominate artery into a common orifice. The tip of the NICP cannula should be just above, but not through, the aortic valve to prevent incompetence. We use an XVIVO॰ dual lumen cannula (central inflow, axially oriented outflow and quick connects for machine circuit connection) for NICP. Silicone tubing is fastened onto the left atrial cuff and placed through the mitral valve to render the valve incompetent and prevent LV distention during NICP.

\section{Cardiopulmonary Bypass Circuit.}

Cardiopulmonary bypass (CPB) circuits are configured based on the weight of the recipient. In $15-25 \mathrm{~kg}$ baboons, we use $1 / 4$ inch $(6.4 \mathrm{~mm})$ and $3 / 8$ inch $(9.5 \mathrm{~mm})$ tubing, for the arterial inflow and venous outflow ends of the circuit, respectively. In baboons less than $15 \mathrm{~kg}$, we elect to use $1 / 4$ inch $(6.4 \mathrm{~mm})$ tubing for both arterial and venous end of the circuit to minimize the dilutional effects of CPB. We use 
centrifugal pump heads along with leukocyte filters and oxygenators as listed in Table 2. Priming volumes are based on the length and size of the circuit tubing and the oxygenator used, but generally around $600 \mathrm{cc}$ of crystalloid is required, and is supplemented with 3,000 units of heparin, $0.5 \mathrm{gm} / \mathrm{kg}$ of mannitol and $50 \mathrm{mEq}$ of sodium bicarbonate. We elect to use retrograde autologous priming of the circuit instead of priming the circuit with blood products. When the heart is reperfused, and prior to weaning $\mathrm{CPB}, 1 \mathrm{mg} / \mathrm{kg}$ of lidocaine and $1 \mathrm{gm}$ of magnesium are administered.

\section{Recipient Heart Explantation and Donor Implantation:}

Heart implantation has been described extensively elsewhere. ${ }^{16,17}$ Here, we will emphasize the implantation process in the context of a pig xenograft donor biatrial implantation into an NHP recipient model. In clinical allotransplantation, studies have shown an advantage of bicaval technique of implantation due to reduced tricuspid and mitral valvular insufficiency, arrythmias and improved atrial geometry. ${ }^{18}$ However, we have seen an improved survival with the biatrial technique performed to avoid caval obstruction from anastomoses twisting, due to donor-recipient malalignment, and caval suture line narrowing. We have not encountered arrythmias and valvular insufficiencies.

The baboon sternum is the particularly thin (close to $0.25 \mathrm{~cm}$ in $10-30 \mathrm{~kg}$ animals). It is important to use fingers to palpate at every rib space and define the midline superiorly and inferiorly to minimize malunion upon chest closure. The sternum is highly malleable, which makes it difficult to perform a midline sternotomy with an oscillating saw. We prefer a bicaval CPB cannulation technique with a right-angled metal tipped cannula in the superior vena cava (SVC) and a straight venous cannula for the inferior vena cava (IVC). Approximate cannula sizes are listed in table 3. The IVC cannula is placed to minimize the risk of IVC stenosis closer to the lateral border of the right atrium, just superior to the cavoatrial junction. We tighten caval umbilical tapes on Rummel sliders at the SVC and IVC cannulation sites.

We do not vent the left side of the xenografts during explantation or implantion. The recipient's cardiectomy is standard. The ventricles are removed from the atria and the great vessels separated close to their outlet valves. We prefer to leave the recipient distal pulmonary artery (PA) long and shorten the donor's instead. This makes the PA-PA anastomosis implant easier to expose and less likely to injure the early branching right main PA.

We fashion a pericardial well from the anteriorly divided sac. The left edge is fastened to silk ligatures placed on snaps. During the left atrial anastomosis, the pleural space can be opened, and the heart can be dropped into the left chest. This setup makes the initial posterior left atrial intima-to-intima suture line to be performed efficiently. The heart is rested on a cold saline-soaked sponge and can be relocated within the pericardium on subsequent anastomoses. The implantation sequence is performed with the left atrial anastomosis, followed by the right atrial septal anastomosis and then the PA and aortic anastomoses. If NICP is performed, we elect to perfuse the xenograft every 15 minutes $^{2}$ The cross-clamp is removed, aorta de-aired with a 18 gauge needle followed by the remainder of the right atrial anastomosis to minimize warm ischemia time. We perform the atrial anastomoses with 4-0 double 
armed prolene and the PA and aorta with 5-0 double armed prolene. The xenograft is defibrillated and temporarily paced as needed upon reperfusion and weaning from cardiopulmonary bypass. When the heart is full, the coronary sinus is examined, as an azygous vein off the coronary sinus exists in pigs. ${ }^{19}$ This can be a major source of bleeding upon cardiac reperfusion.

A telemetry device (DSI L21, New Brighton, MN, USA) is placed in the subxiphoid preperitoneal space. One pressure transducer is placed in the aorta within the aortic cannula site after decannulation, the other pressure transducer is placed in the right atrial appendage. The electrocardiogram (EKG) lead is placed on the anterior surface of the right atrial appendage and the ground lead is placed on the left ventricle near the apex. Up to 8 simultaneous implants can be transduced through one receiver (figure 2). Chest tubes are placed in the bilateral pleural spaces and the pericardial well, connected to a Jackson-Pratt bulb (table 2). The sternum is closed with 0-ethibond suture in an interrupted fashion. Fascial and skin closure is performed in several layers, taking special care to enclose the telemetry hardware in a separate fascial pouch in the subxiphoid space. A post-operative chest $x$-ray is performed to ensure there are no large undrained pleural effusions or pneumothoraces.

\section{Immediate Postoperative Management (first 48 hours)}

We have developed a post-operative protocol after extensive anecdotal experience with this model (table 4). Following chest closure, we make every attempt at extubation within 2-3 hours. If prolonged intubation is required, the recipient should remain sedated with fentanyl $2-4 \mathrm{mcg} / \mathrm{kg} / \mathrm{hr}$ and propofol $1-4 \mathrm{mg} / \mathrm{kg} / \mathrm{hr}$. Caution should be used with fentanyl boluses, as even at small doses, they can cause hypotension. Sevoflurane with a minimum alveolar concentration (MAC) of 1.0-1.2 works well with fentanyl/propofol but may have some cardiac depressive effects. Once the recipient is extubated, it is transferred to our intensive care unit (ICU) with specialized climate-controlled cages that can administer a fractional inspired oxygen concentration of up to $40 \%$ (figure 1A). It also has a tether system that allows for tunneling of the central line through a jacket and the cage wall onto a manifold that can be connected to medication administration pumps (figures 1B-D). Any postoperative drips and fluids can be administered, and frequent blood sampling can be employed. Our preference is to begin fluids at 1.5 times the basal rate by ideal body weight and titrate up and down judiciously in the first few hours after transplantation based on fluid and acid/base status and the amount of resuscitation needed. We also empirically load the recipient with $1 \mathrm{mg} / \mathrm{kg}$ of intravenous lidocaine intraoperatively and start an amiodarone drip at 1 $\mathrm{mcg} / \mathrm{kg} / \mathrm{min}$, since cardiac xenografts are prone to arrythmias. Antiarrhythmics are weaned in a staged fashion if the animal demonstrates minimal to no ectopy in the first 12 hours postoperatively. If ventricular arrythmias occur despite these measures, lidocaine is preferred as the porcine xenograft responds to this better than amiodarone in this setting. It can be given as an additional $1 \mathrm{mg} / \mathrm{kg}$ bolus, followed by a $20 \mathrm{mcg} / \mathrm{kg} / \mathrm{min}$ infusion. Defibrillator paddles are kept on the recipient animal, underneath the jacket for the first 48 hours after transplantation with a defibrillator setup cage-side for swift access in the event of ventricular fibrillation (figure 1E). In our experience these paddles were rarely used but saves time if defibrillation is required in jacketed animals. 
Recipients of xenografts from swine are particularly susceptible to hypocalcemia, for reasons that are not understood. Therefore, calcium should be expected to be repleted at a rate of about $500 \mathrm{mg}$ to $1 \mathrm{~g}$ of calcium gluconate every hour, driven by ionized calcium levels (goal ionized calcium levels are 1.0-1.2 $\mathrm{mg} / \mathrm{dL}$, see table 4). Potassium is repleted for levels below $3.2 \mathrm{mEq} / \mathrm{dL}$ at $1 \mathrm{mEq} / \mathrm{kg}$ infused in $50 \mathrm{cc}$ of saline over 1 hour, up to $20 \mathrm{mEq}$. Bicarbonate is repleted only in severe cases of metabolic acidosis in the absence of hypercarbic respiratory insufficiency. The bicarbonate deficit is calculated by the following formula: $1 / 3 \times$ weight $(\mathrm{kg}) \times$ base deficit. The deficit is repleted by $50 \%$ and a blood gas is repeated in 1 hour to measure response. It should be noted that the cardiac xenograft can exhibit marked sinus tachycardia, but is left untreated, with heart rates of $148 \pm 14$ beats per minute in our most recent cohort. ${ }^{4}$ Moreover, common electrocardiographic morphologies are noted in figure 2 and should be considered "normal variants" of a sinus rhythm.

We have protocolized sedating and examining recipients each morning in the first 48 hours postoperatively to assess cardiopulmonary and volume status more appropriately and to manage chest tubes. While there is a telemetry pressure transducer is placed in the right atrial appendage, central venous pressure (CVP) is somewhat unreliable in an awake, non-sedated and ambulatory baboon transplant recipient. Therefore, volume status is assessed based on chest X-ray, ultrasound and physical exam. We examine the xenograft by echocardiography, the lungs by portable chest $x$-ray and strip the chest tubes and let them drain the pleural spaces entirely. The chest tubes remain until total pleural fluid from each drain is less than $50 \mathrm{cc}$ in a 24-hour period. Generally, if more than $100 \mathrm{cc}$ of fluid is collected total, the drains will stay collectively for another 24 hours, until the tubes can be reexamined and a repeat x-ray can be performed. Generally, our recipients can be transferred out of the ICU the morning of postoperative day two and by that time they are typically off any supplemental medications, drips or fluids and are receiving only maintenance immunosuppression. Anticoagulation is started at 50 units per hour and then titrated slowly to an ACT of twice baseline when surgically appropriate, usually by postoperative day five.

\section{Long-term Postoperative Management and Graft Surveillance:}

During the first two weeks postoperatively, animals are being monitored by continuously by cameras and telemetry. The telemetry readings sometime raise false alarms due to loss of connection with the receiver and the baboon status is confirmed by observing them on the camera. Both cameras and telemetry readings can be accessed remotely and is also recorded. The laboratory examination is performed 1-2 times per week and then twice monthly for the first month. Thereafter, laboratory examination is done every month and reserved for clinical status changes. Standard laboratory examination includes a complete blood count (CBC), complete metabolic panel (CMP), activated clotting time (ACT) and a troponin. Recipients are visually examined daily for behavior, appetite, abdominal distention, respiratory effort, global and dependent edema and for normal stool and urine production. Cage and jacket changes are done every 2 weeks and in doing so, the animal is sedated and a full physical exam is performed. Special attention is given to the animal's weight, body condition, surgical incision, central line exit site, 
mucosal membranes and abdomen. An echocardiographic examination is also performed in parasternal short, parasternal long and 4-chamber views (figures 3A-D).

In addition to telemetric and echocardiographic data, we have started to employ endomyocardial biopsies, right heart catheterizations and coronary angiograms to our surveillance regimen through femoral venous and arterial access sites. While the specifics on how to perform these procedures are not detailed here, the supplies required to perform these procedures specify for cardiac xenotransplantation are detailed in table 5. Our preference is to perform these surveillance adjuncts every 2 months starting 30 days after transplantation.

\section{Common Problems in Pig-to-Baboon Cardiac Xenotransplantation:}

The tunneled central line, jacket and associated tether system are the most common problems in this baboon model. All animals are acclimated to the system 1 week prior to the placement of a central line and animals that show signs of stress, intolerance to the system or otherwise incapable of keeping their jacket on and intact during this time frame are excluded from study. Despite this, however, these animals have insatiable curiosity and are continuously puling and jerking the tether. For the tether to function properly several joints have to be properly secured. The transplanted baboons, due to continuous activity and manipulation of tether, make these joints malfunction which can result in supercoiling of the tether, damaging of the central line and repeated manipulation of the line which can result in infection. Each of the following are addressed below-

1. Supercoiling tethers: The tether system is depicted in figures $1 \mathrm{~B}$ and $1 \mathrm{C}$, which consists of a tunneled catheter, protected by a jacket worn by the recipient, thread through a metal tether system that houses the catheter and 4Fr silicon intravenous (IV) tubing. The IV tubing connects through a swivel, to the outside of the cage with a multi-channel pump system for drug administration and blood draws. The tether system is predicated on the ability to have one fixed end (closest to the jacket) and one end attached to a swivel. Therefore, any rotation of the IV line within the tether is prevented with axial tension created by the beginning of a supercoil. However, when this system fails, axial rotation of the IV line within the tether is created and a supercoil develops with the recipient's free movement around the cage system (figure 4A). This supercoil can cause an obstruction or can lead to being pulled off from its attachment at the either end. The most common indication that is unique to supercoiling is the inability to either flush the line or draw back blood. This can largely be prevented by daily visual inspection of the swivel and ensuring that it can freely rotate around its axis with minimal interference. If a supercoil does occur, the animal must be sedated and the entire line and tether system must be replaced in a sterile fashion.

2. Damage to the central line: NHPs can gain access to the central line (or other devices such as power or pump cords) by means of either damaging the pouch on the back of the jacket, which houses the infusion ports of the central line, or by disconnecting the metal tether from the swivel and exposing the silicon IV tubing (figures 4B and $4 \mathrm{C}$ ). In animals that have demonstrated the ability and desire to disconnect the tether, we reinforce the distal and proximal end of the tether with steel (figure 4D). 
3. Central line infections: central lines are the most common sources of infection with our recipients and are difficult to treat. Moreover, these animals are all severely immunosuppressed and require daily medication administration, precluding any ability to perform line holidays or line removal without immediate replacement. Therefore, line manipulation should be minimized and if required, sterility is paramount. We also have developed a protocol for prophylaxis, which consists of an antibiotic and heparin lock for any line manipulations, including animal transport, cage changes or catheter/tether repair. Formulations are based on historic bacterial susceptibility and previously literature on this topic. ${ }^{20,21}$ Any recipient with a fever (indicated by elevated temperature readings on the telemetry) and outside of the immediate postoperative phase is cultured, started on broad spectrum antibiotics and narrowed based on the presence or absence of cultured pathogen. Definitive antibiotic selection and course is based on the pathogen isolated, likelihood of biofilm formation and prior antibiotic course failures. If there is damage to the catheter, there are repair options. However, the central line is sometimes opted to be replaced entirely, preferably after clearance of bacteremia if logistically feasible. Venous access can be obtained on the contralateral internal jugular vein or either external jugular vein. One vein of either internal or external jugular vein should be preserved out of the four main sources of drainage of the neck to ensure proper drainage of the head.

4. Blockage of central line due to precipitation of infused drugs: Some of the infused drugs in our regimen are incompatible with heparinized saline. This incompatibility leads to precipitation of these drugs in the IV tubing when infusing concurrently. We routinely reconstitute all of our drugs in $5 \%$ dextrose solution, which prevents drug precipitation.

5. Central line dislodgement: A very rare complication is when the central catheter is either pulled out of the internal jugular vein or starts leaking at the site of insertion. The animal in this case demonstrates accumulation of fluids in the cheek pouch. Definite diagnosis is made via fluoroscopy when the dye is seen leaking around the insertion site of the catheter. This catheter requires immediate replacement.

\section{Nutritional supplementation:}

Weight loss does occur in most recipients after transplantation. Our IACUC regulations necessitate $20 \%$ weight loss from the time of animal study enrollment as an endpoint requiring euthanasia, regardless of clinical status of the animal. Therefore, we have protocolized our care of these animals to minimize weight loss.

All animals are placed on a high protein diet with Teklad Global high protein biscuits (Inotiv, Inc., West Lafayette, IN, USA) and given diet enrichment of high calorie snacks, to maintain body weight after transplantation. Animals are monitored for biscuit intake daily, with a goal target caloric intake of 25 $\mathrm{kcal} / \mathrm{kg} /$ day and total protein intake of $1 \mathrm{~g} /$ day. If an animal reaches threshold of $10 \%$ weight loss, appetite stimulants are administered, and potential causes of weight loss and decreased appetite are 
investigated. If an animal reaches a threshold of $15 \%$ weight loss, intermittent bolus tube feeds twice weekly are administered by gavage feeding to supplement nutrition.

\section{Results And Discussion:}

We have developed a protocolized approach to performing cardiac xenotransplantation of genetically modified xenografts in a non-human primate recipient model culminating from years of experience. It requires a multidisciplinary team of scientists, immunologists, clinical staff, surgeons and perfusionists. Moreover, the complexity of this model requires expertise and experience in working with non-human primates and heart transplant recipients. However, this is a robust pre-clinical model of cardiac xenotransplantation with immediate translational potential even if appropriate FDA approval is required. ${ }^{2-26}$ Moreover, postoperative protocolization, while specifically optimized for cardiac xenotransplantation, can be applied to cardiac allotransplantation and other disciplines of transplantation using NHP models.

This serves as a model for life-supporting, orthotopic transplantation that emulates clinical heart allotransplantation and has afforded opportunity to examine several outstanding questions in cardiac xenotransplantation. Längin and colleagues elegantly demonstrated that non-ischemic continuous preservation (NICP) can overcome PCXD almost universally, which was previously an insurmountable barrier to clinical translation. ${ }^{2,13,14}$ We have demonstrated that alternative strategies to NICP that minimize ischemia reperfusion injury may warrant further investigation and signal toward a potential underlying mechanism of PCXD. ${ }^{27}$ NICP has also afforded our group the opportunity to use this model to assess the progressive and iterative genetic modifications of cardiac xenograft donors and their effect on improving overall xenograft survival. ${ }^{3}$ We have demonstrated that select "multi-gene" cardiac xenografts, with multiple knockouts and human transgene expression of complement regulatory, thromboregulatory and anti-inflammatory proteins have preserved life-supporting function up to 9 months. We have also demonstrated that physiologic mismatch is present in xenografts after transplantation and that posttransplantation xenograft growth can be reduced with growth hormone receptor knockout donors. ${ }^{4}$ Current studies are focused on understanding contributors of life-limiting post-transplantation xenograft growth in this model.

\section{Conclusion:}

The pig-to-baboon model of cardiac xenotransplantation continues to be a challenging, but prototypic model for demonstrating preclinical efficacy for the use of cardiac xenografts from swine for end-stage heart failure, without the ability to obtain an allograft or durable mechanical circulatory support. Specific keys to success include a protocolized approach to the procurement of the xenograft, implantation and postoperative care of the animal in the acute and long-term phases of care. Many questions remain to characterize cardiac xenografts in the foreign environment of a xenogeneic recipient, and surveillance measures will also be a key to demonstrating pre-clinical efficacy prior to clinical translation, which have 
been summarized here as well. Moreover, once clinical translation is realized, principles of cardiac xenograft care and surveillance here can be used as a template for the eventual human application of this novel source of solid organs for transplantation in patients with failing hearts and no alternatives.

\section{Declarations:}

\section{Competing Interests:}

Dave Ayares is employed by Revivicor, Inc., a subsidiary of United Therapeutics. United Therapeutics was not involved in the study design, collection, analysis, interpretation of data, the writing of this article or the decision to submit it for publication. The remaining authors declare that the research was conducted in the absence of any commercial or financial relationships that could be construed as a potential conflict of interest.

\section{Funding:}

This study was supported by funding from United Therapeutics, Inc and the NIAID, NIH grant 5U19090959-10. David Ayares is an employee of Revivicor, Inc. There are no conflicts of interest to disclose by any other authors.

\section{References:}

1. Bui AL, Horwich TB, Fonarow GC. Epidemiology and risk profile of heart failure. Nat Rev Cardiol. 2011;8(1):30-41. doi:10.1038/nrcardio.2010.165

2. Längin $M$, Mayr T, Reichart $B$, et al. Consistent success in life-supporting porcine cardiac xenotransplantation. Nature. 2018;564(7736):430-433. doi:10.1038/s41586-018-0765-z

3. Mohiuddin M, Goerlich C, Singh A, et al. Progressive Genetic Modifications of Porcine Cardiac Xenografts Extend Survival to 9 Months. In Review; 2021. doi:10.21203/rs.3.rs-382870/v1

4. Goerlich CE, Griffith B, Hanna P, et al. The Growth of Xenotransplanted Hearts Can Be Reduced with Growth Hormone Receptor Knockout Pig Donors. J Thorac Cardiovasc Surg. Published online September 2021. doi:10.1016/j.jtcvs.2021.07.051

5. Reichart B, Längin M, Denner J, Schwinzer R, Cowan PJ, Wolf E. Pathways to Clinical Cardiac Xenotransplantation. Transplantation. 2021;105(9):1930-1943. doi:10.1097/TP.0000000000003588

6. Rabin R. In a First, Surgeons Attached a Pig Kidney to a Human, and It Worked. The New York Times. https://www.nytimes.com/2021/10/19/health/kidney-transplant-pig-human.html. Published October 21, 2021.

7. Cooper DKC, Keogh AM, Brink J, et al. Report of the xenotransplantation advisory committee of the international society for heart and lung transplantation: J Heart Lung Transplant. 2000;19(12):11251165. doi:10.1016/S1053-2498(00)00224-2 
8. U.S. Food and Drug Adminstration. Source Animal, Product, Preclinical, and Clinical Issues Concerning the Use of Xenotransplantation Products in Humans. Published online December 2016.

9. Percie du Sert N, Hurst V, Ahluwalia A, et al. The ARRIVE guidelines 2.0: updated guidelines for reporting animal research. BMJ Open Sci. 2020;4(1):e100115. doi:10.1136/bmjos-2020-100115

10. Azimzadeh AM, Byrne GW, Ezzelarab M, et al. Development of a consensus protocol to quantify primate anti-non-Gal xenoreactive antibodies using pig aortic endothelial cells. Xenotransplantation. 2014;21(6):555-566. doi:10.1111/xen.12125

11. Goerlich CE, DiChiacchio L, Zhang T, et al. Heterotopic Porcine Cardiac Xenotransplantation in the Intra-Abdominal Position in a Non-Human Primate Model. Sci Rep. 2020;10(1):10709. doi:10.1038/s41598-020-66430-x

12. Copeland H, Hayanga JWA, Neyrinck A, et al. Donor heart and lung procurement: A consensus statement. J Heart Lung Transplant. 2020;39(6):501-517. doi:10.1016/j.healun.2020.03.020

13. Längin M, Reichart B, Steen S, et al. Cold non-ischemic heart preservation with continuous perfusion prevents early graft failure in orthotopic pig-to-baboon xenotransplantation. Xenotransplantation. 2021;28(1). doi:10.1111/xen.12636

14. DiChiacchio L, Singh AK, Lewis B, et al. Early Experience With Preclinical Perioperative Cardiac Xenograft Dysfunction in a Single Program. Ann Thorac Surg. 2020;109(5):1357-1361. doi:10.1016/j.athoracsur.2019.08.090

15. Goerlich CE, Griffith B, Singh AK, et al. Blood Cardioplegia Induction, Perfusion Storage and Graft Dysfunction in Cardiac Xenotransplantation. Front Immunol. 2021;12:667093. doi:10.3389/fimmu.2021.667093

16. Trento A, Czer LS, Blanche C. Surgical techniques for cardiac transplantation. Semin Thorac Cardiovasc Surg. 1996;8(2):126-132.

17. Shumway NE, Lower RR, Stofer RC. Transplantation of the heart. Adv Surg. 1966;2:265-284.

18. Morgan JA, Edwards NM. Orthotopic cardiac transplantation: comparison of outcome using biatrial, bicaval, and total techniques. J Card Surg. 2005;20(1):102-106. doi:10.1111/j.08860440.2005.05011.x

19. Wingerd BD. Pig Anatomy and Dissection Guide. Bluedoor; 2006.

20. Droste JC. Stability and in vitro efficacy of antibiotic-heparin lock solutions potentially useful for treatment of central venous catheter-related sepsis. J Antimicrob Chemother. 2003;51(4):849-855. doi:10.1093/jac/dkg179

21. Robinson JL, Tawfik G, Saxinger L, Stang L, Etches W, Lee B. Stability of heparin and physical compatibility of heparin/antibiotic solutions in concentrations appropriate for antibiotic lock therapy. J Antimicrob Chemother. 2005;56(5):951-953. doi:10.1093/jac/dki311

22. Goerlich CE, Chan JL, Mohiuddin MM. Regulatory barriers to xenotransplantation. Curr Opin Organ Transplant. 2019;24(5):522-526. doi:10.1097/MOT.0000000000000678 
23. Hawthorne WJ, Cowan PJ, Bühler LH, et al. Third WHO Global Consultation on Regulatory Requirements for Xenotransplantation Clinical Trials, Changsha, Hunan, China December 12-14, 2018: "The 2018 Changsha Communiqué" The 10-Year Anniversary of The International Consultation on Xenotransplantation. Xenotransplantation. 2019;26(2):e12513. doi:10.1111/xen.12513

24. Pierson RN, Fishman JA, Lewis GD, et al. Progress Toward Cardiac Xenotransplantation. Circulation. 2020;142(14):1389-1398. doi:10.1161/CIRCULATIONAHA.120.048186

25. Cooper DKC, Pierson RN, Hering BJ, et al. Regulation of Clinical Xenotransplantation-Time for a Reappraisal. Transplantation. 2017;101(8):1766-1769. doi:10.1097/TP.0000000000001683

26. Platt JL, Piedrahita JA, Cascalho M. Clinical xenotransplantation of the heart: At the watershed. $J$ Heart Lung Transplant. 2020;39(8):758-760. doi:10.1016/j.healun.2020.06.002

27. Goerlich CE, Griffith B, Singh AK, et al. Blood Cardioplegia Induction, Perfusion Storage and Graft Dysfunction in Cardiac Xenotransplantation. Front Immunol. 2021;12:667093. doi:10.3389/fimmu.2021.667093

\section{Tables:}

Table 1. Orthotopic Heart Procurement Checklist 


\section{Essential back-table supplies:}

Suction tubing and yankauer suction tip $\mathrm{x} 2$

Rummel torniquet $\mathrm{x} 2$

Aortic cardioplegia cannula (9Fr) and cannula bladder irrigation tubing

Aortic vascular clamp, straight vascular clamp $\times 2$

Internal paddles

Saline slush

\section{Preparation of Donor for OHTx Procurement:}

Shave: chest, abdomen, neck, back and arms

Access: peripheral IV at ear; alternatively, central line can be placed in groin or neck

Airway: tracheostomy preferred over oral airway

Arterial Line: femoral percutaneous or surgically placed carotid (if doing tracheostomy)

Prep field: betadine scrub followed by chlorohexidine scrub, from neck to groin and as far laterally as possible

Setup: Bovie electrocautery, bovie grounding pad on back, suction x2, internal paddles connected and ready

Extra hand for back table

\section{OHTx Procurement:}

Midline sternotomy, Pericardial well creation

Dissect Great Vessels

Clear Aorta, SVC/IVC in order for vascular clamps to be placed

Place aortic cardioplegia cannula

Obtain $25 \%$ of $30 \mathrm{cc} / \mathrm{kg}$ fresh donor blood and mix with cardioplegia on ice (if using blood cardioplegia)

Prime cardioplegia tubing and de-air

Administer Cardioplegia $(30 \mathrm{cc} / \mathrm{kg}$ ) through pressure bag and tubing when ready for cardioectomy

Vent left and right sides of heart if distention observed

Table 2. Orthotopic Cardiac Xenotransplantation - Important Materials 


\section{Name}

Manufacturer

\section{Donor:}

9 Fr Cardioplegia Aortic Root Cannula

Bladder Irrigation Tubing for Cardioplegia

Extension IV Tubing Set

Recipient:

10 Fr Hickmann Tunneled Triple Lumen Catheter

Data Sciences International (DSI) Telemetry Device L21

Chest Tube - Perforated Flat Drain

Medline (USA)

Medline (USA)

Medline (USA)

BARD (USA)

606560

DSI (USA)

DSI L21

Chest Tube - Perforated Round Drain x2

Jackson-Pratt Bulb Reservoir

Cardiopulmonary Bypass Circuit:

Centrifugal Perfusion Pump

Cardiopulmonary Bypass Oxygenator $(<15 \mathrm{~kg}$ animal)

Cardiopulmonary Bypass Oxygenator (15-15 kg animal)

Cardiopulmonary Bypass Leukocyte Filter
Sorin Revolution (London, 050300000 UK)

Terumo Cardiovascular (USA)

Terumo Cardiovascular (USA)

Terumo Cardiovascular (USA)
2C4041

$12656-28$
Reference

Number

DYNJWE1310

DYNJWE1323A

DYNJWE1305 


\section{Perioperative Anesthesia and Sedation:}

Early extubation when able

If prolonged intubation required:

Fentanyl 2-4mcg/kg/hr, propofol 2-8 ml/hr (20-80 mg/hr or 1-4 mg/kg/hr). Max propofol dose = $6 \mathrm{mg} / \mathrm{kg} / \mathrm{hr}$.

Sevoflurane works well with fentanyl/propofol but may have some cardiac depressive effects

Administer sevoflurane for a MAC goal of 1.0-1.2

\section{Electrolytes:}

Calcium should be expected to be repleted at a rate of approximately $500 \mathrm{mg}-1 \mathrm{~g}$ per hour

iCal goal-driven: 1.0-1.2; if calcium $<1.0,1 \mathrm{~g}$ calcium gluconate, if $1.0<1.2,500 \mathrm{mg}$

Potassium, replete at $1 \mathrm{mEq} / \mathrm{kg}$ repletion

Replete if potassium $\leq 3.2$

\section{Acid/Base (starting algorithm):}

"Bicarb Deficit" (in mEq of Bicarb) $=1 / 3 \times$ baboon weight in $\mathrm{kg} \times$ Base Deficit

Replete half of base deficit, re-check arterial blood gas in 1 hour to measure response

\section{Anti-arrhythmic:}

Amiodarone: $5 \mathrm{mg} / \mathrm{kg} \times 3$ if needed ( $\mathrm{max} 15 \mathrm{mg} / \mathrm{kg}$ load for acute treatment)

Lidocaine: Loading dose of $1 \mathrm{mg} / \mathrm{kg}$, IV Infusion: $20 \mathrm{mcg} / \mathrm{kg} / \mathrm{min}$ (not to be exceeded in patients with heart failure)

Table 5. Supplies for Endomyocardial Biopsy, Right Heart Catheterization, and Coronary Angiography 


\begin{tabular}{|lll|}
\hline Name & Manufacturer & $\begin{array}{l}\text { Reference } \\
\text { Number }\end{array}$ \\
\hline $\begin{array}{l}\text { Arrow Single Lumen Balloon Wedge-Pressure } \\
\text { Catheter }\end{array}$ & Teleflex (Ireland) & Al-07125 \\
\hline 4 Fr JL 1.5 Performa Angiographic Catheter & Merit Medical (USA) & $7440-82$ \\
\hline 6 Fr Impulse LCB & Boston Scientific (USA) & H749165991950 \\
\hline 5 Fr Impulse FL3.5 & Boston Scientific (USA) & H74916391211 \\
\hline 6 Fr Impulse FR3.5 & Boston Scientific (USA) & H74916599010 \\
\hline 5 Fr Impulse IM & Boston Scientific (USA) & H749163912011 \\
\hline Endomyocardial Biopsy Forcep & $\begin{array}{l}\text { Argon Medical Devices } \\
\text { (USA) }\end{array}$ & 190051 \\
\hline
\end{tabular}

\section{Figures}



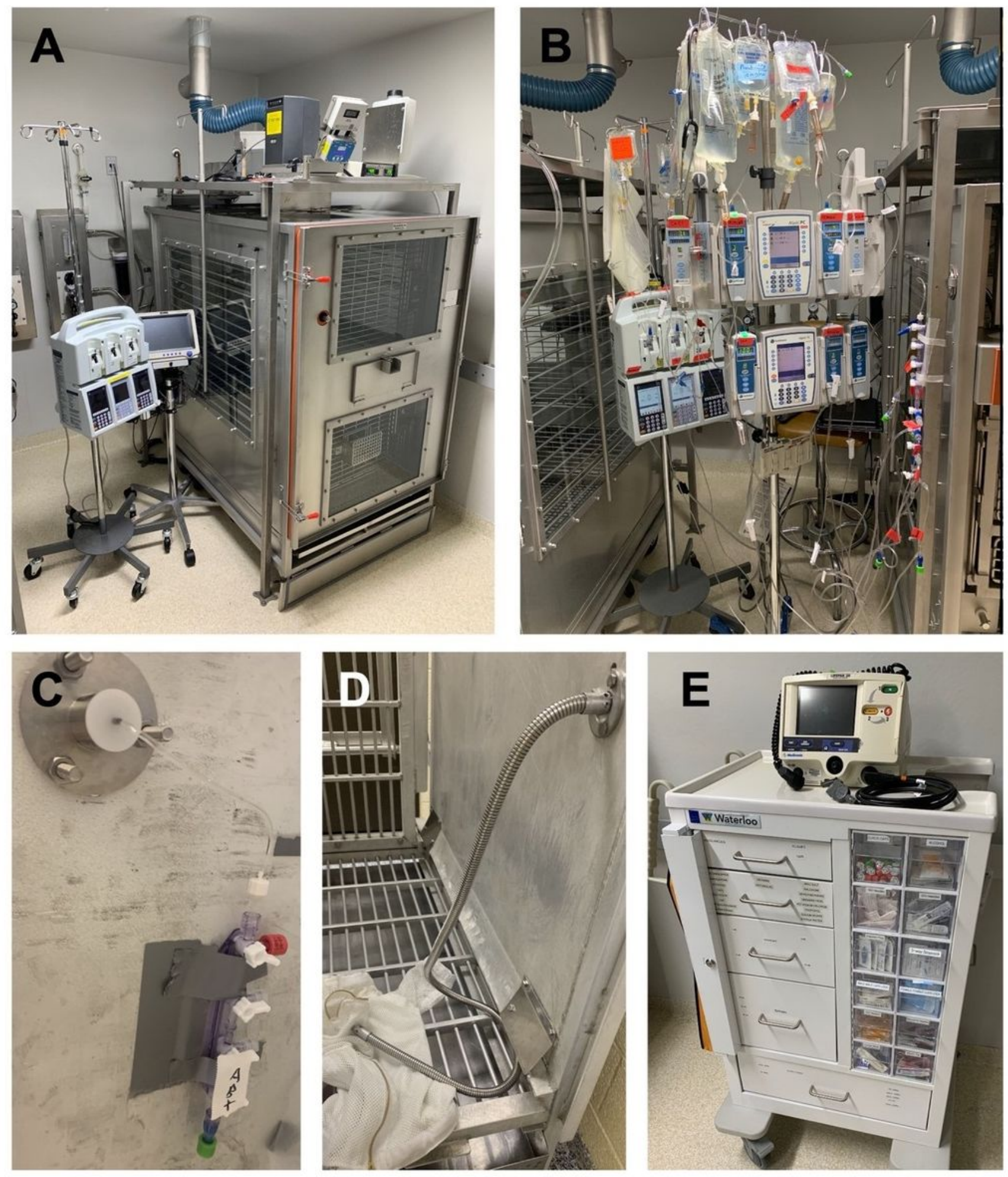

\section{Figure 1}

Intensive Care Unit (ICU) Setup. A) Climate controlled ICU cage with high-efficiency particulate air (HEPA) filtration, ability to provide ambient oxygen saturation of up to $40 \%$ fractional inspiration of inspired oxygen (FiO2), and a urine collection system B) Example of IV catheter setup with numerous pumps delivering medications $\mathrm{C}$ ) Example of a manifold on the external wall of cage, which connect IV medication delivery pumps to the tether system D) recipient jacket connected to the tether with swivel 
allowing delivery of medication without supercoiling E) ICU cart allowing rapid access to frequently used medications, supplies, and external defibrillator

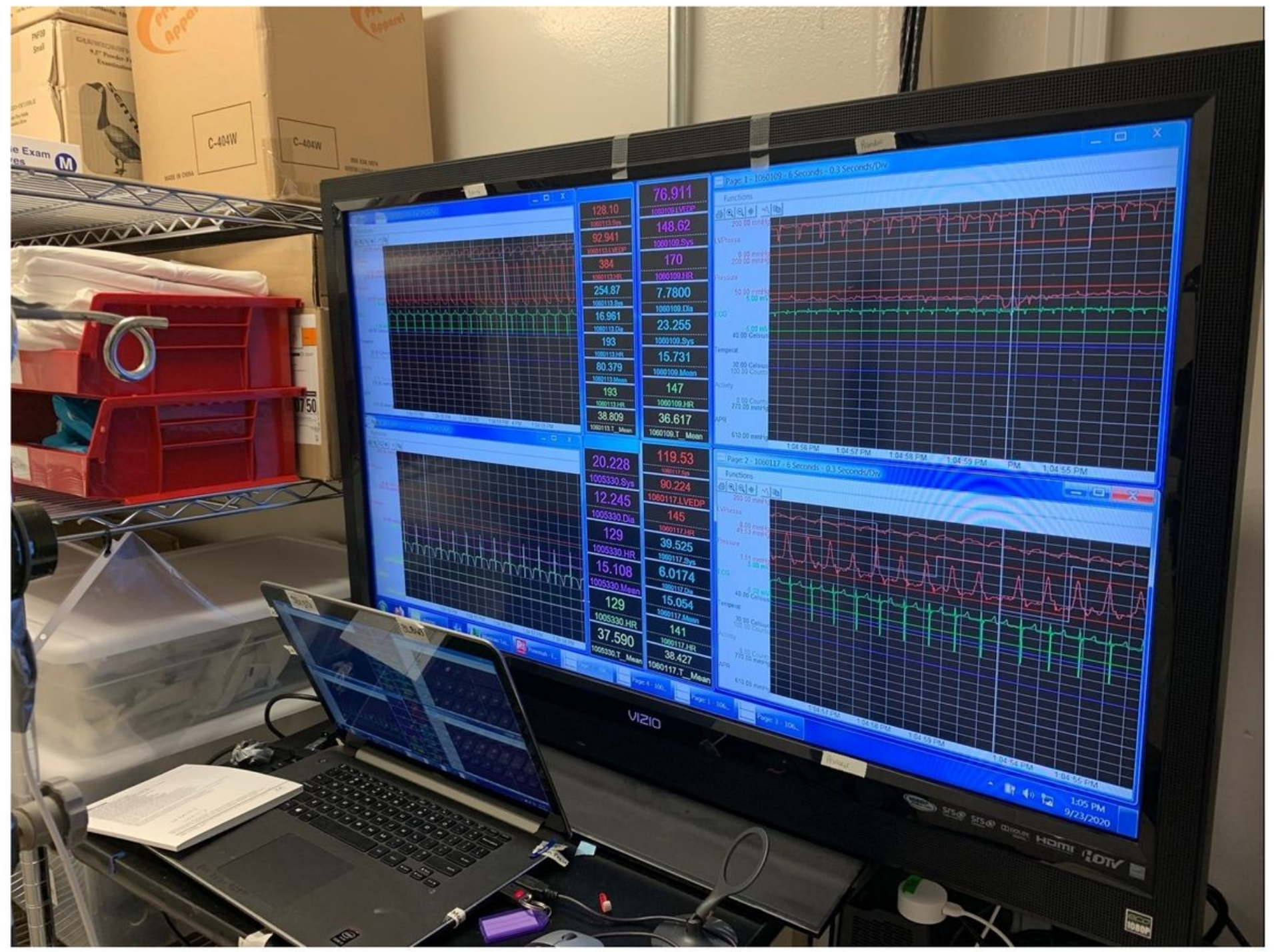

\section{Figure 2}

Electrocardiographic Tracings of Four Simultaneous Postoperative Xenotransplantation Recipients. Realtime invasive telemetric monitoring using the implantable Data Sciences International (DSI) Telemetry Device L21. 

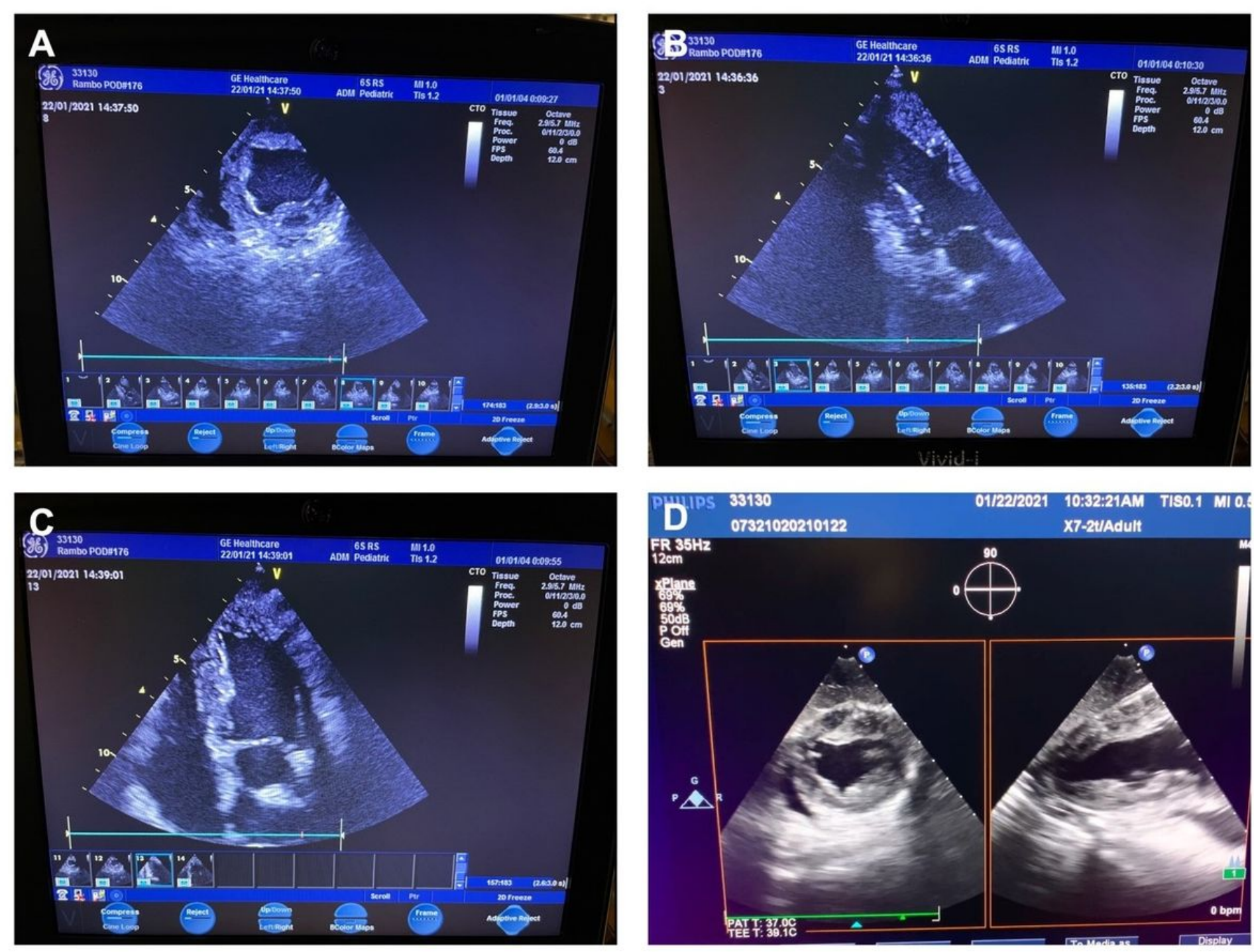

\section{Figure 3}

Echocardiographic Images in Multiple Views. A) Transthoracic echocardiographic (TTE) parasternal short axis view B) TTE parasternal long axis view C) TTE four chamber view D) Transesophageal echocardiographic (TEE) short and long axis views 

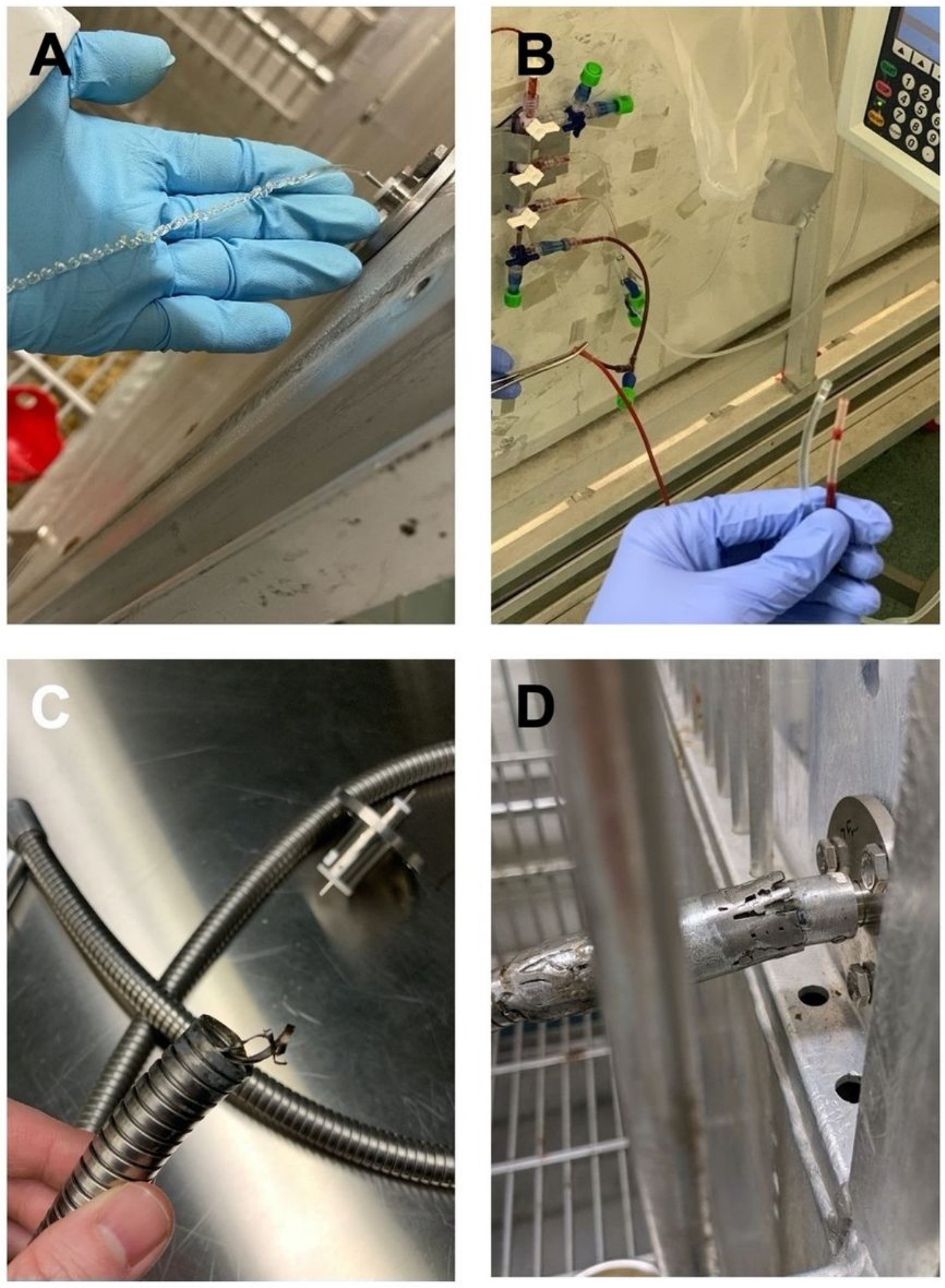

\section{Figure 4}

Potential IV line and tether complications. A) Supercoiling of silicone IV line caused by fixation of tether B) An IV line that after a recipient was able to reach it, causing complete transection, bleeding and ultimately a central line infection C) A tether that was damaged, despite reinforcement with steel, causing the silicon IV tubing to be disconnected from the swivel and a contaminated line D) Reinforced tether to prevent damage 